\title{
Continuous Flow Reactors from Microfluidic Compartmentalization of Enzymes within Inorganic Microparticles
}

Tuuli A. Hakala Friedrich Bialas ${ }^{+}$Zenon Toprakcioglu, Birgit Bräuer, Kevin N. Baumann, Aviad Levin, Gonçalo. J. L. Bernardes, ${ }^{*}$ Christian F. W. Becker,${ }^{*}$ and Tuomas P. J. Knowles*

Department of Chemistry, University of Cambridge, Lensfield Road, Cambridge, CB2 1EW, United Kingdom

${ }^{\perp}$ Institute of Biological Chemistry, Faculty of Chemistry, University of Vienna, Währinger Str.38, 1090, Vienna, Austria

§Instituto de Medicina Molecular, Faculdade de Medicina de Universidad de Lisboa, 1649-028

Lisboa, Portugal

${ }^{\circ}$ Cavendish Laboratory, University of Cambridge, J. J. Thomson Avenue, CB3 0HE Cambridge, United Kingdom

$\ddagger$ Contributed equally to this work

Correspondence should be addressed to:

gb453@cam.ac.uk (G.J.L.B.); christian.becker@univie.ac.at (C.F.W.B,); tpjk2@,cam.ac.uk (T.P.J.K.)

\section{Table of Contents}

Materials

$\mathrm{S} 2$

Supporting Figures and Methods 


\section{Materials}

Bovine serum albumin (\#A7906), $\beta$-galactosidase (\#G3153), D-(+)-Glucose(\#G8270), Fluorescein isothiocyanate isomer I (F7250), 1H,1H,2H,2H-Perfluoro-1-octanol (\#370533), Horseradish peroxidase (\#7732), N,N-Diisopropylethylamine (\#D125806), Piperidin(\#104094), Proteinase K from Tritirachium album (\#P6556) and Sodium Phosphate were obtained from Sigma Aldrich. Fmoc$\mathrm{L}-\mathrm{Arg}(\mathrm{Pbf})-\mathrm{OH}(\# \mathrm{FSC} 1010.0020)$ was purchased from Iris Biotech and FmocL-Asp(OtBu)-OH (\#852005), Fmoc-L-Ser(tBu)-OH (\#852019), Fmoc-L-Leu-OH (\#852011), Fmoc-Gly-OH (\#852001), Fmoc-L-Tyr(tBu)-OH(\#852020), Fmoc-L-Lys(Boc)-OH (\#852012) from Novabiochem. Fmoc-L-Ile$\mathrm{OH}(\# \mathrm{~B} 21154.14) \quad$ from $\quad$ VWR. 2-(1H-Benzotriazol-1-yl)-1,1,3,3tetramethyluronium hexafluorophosphate(\#RL-1030.1000) from Iris Biotech. Resorufin-D-Galactopyranoside (\# R1159), Amplex Red (\#A22177), and Tetramethyl orthosilicate, 99\%, (\#AC203820250) from Thermo Fisher Scientific. Glucose oxidase (\#195196) was purchased from MP biomedicals. 


\section{Supporting Figures and Methods}

\section{Analytic data from R5 peptide synthesis}

a

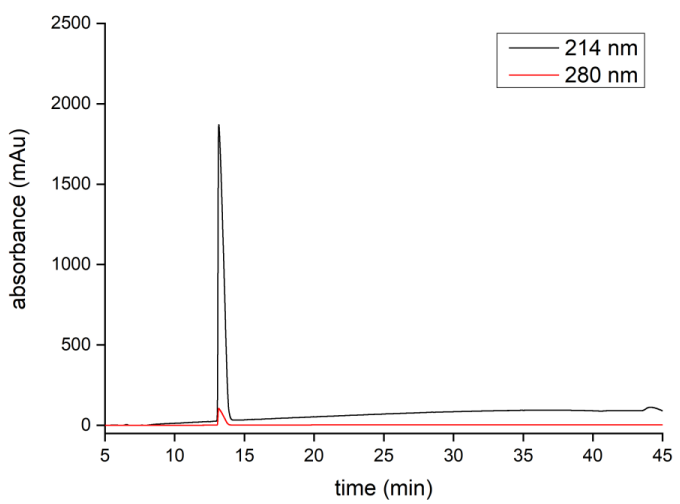

c

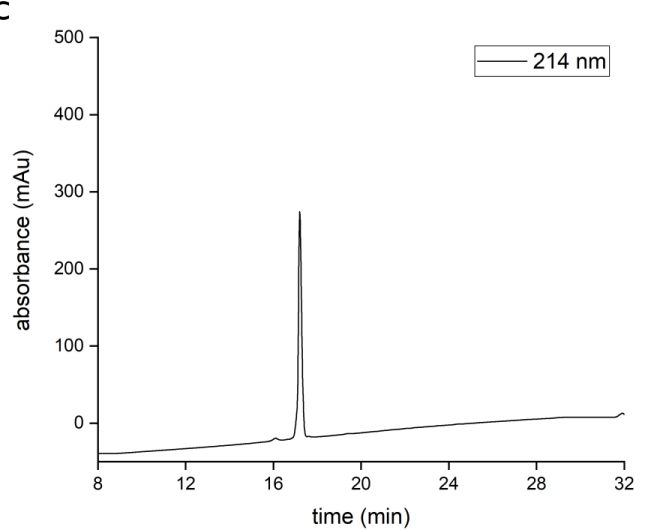

b

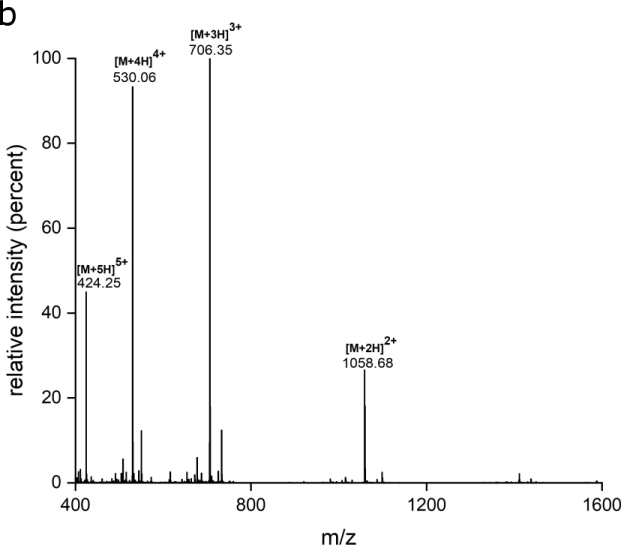

d

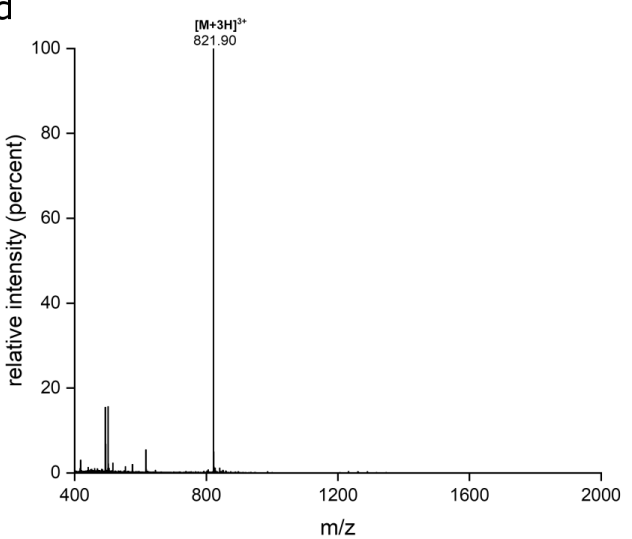

Figure S1: R5 peptide analytics. A reverse-phase HPLC chromatogram (a and c) and direct injection mass spectrum (DI-MS) (b and d) to show the purity of the R5 (a and b) and Cy5-R5 (c and d) peptides. 


\section{SEM of silica microparticle cross-section}

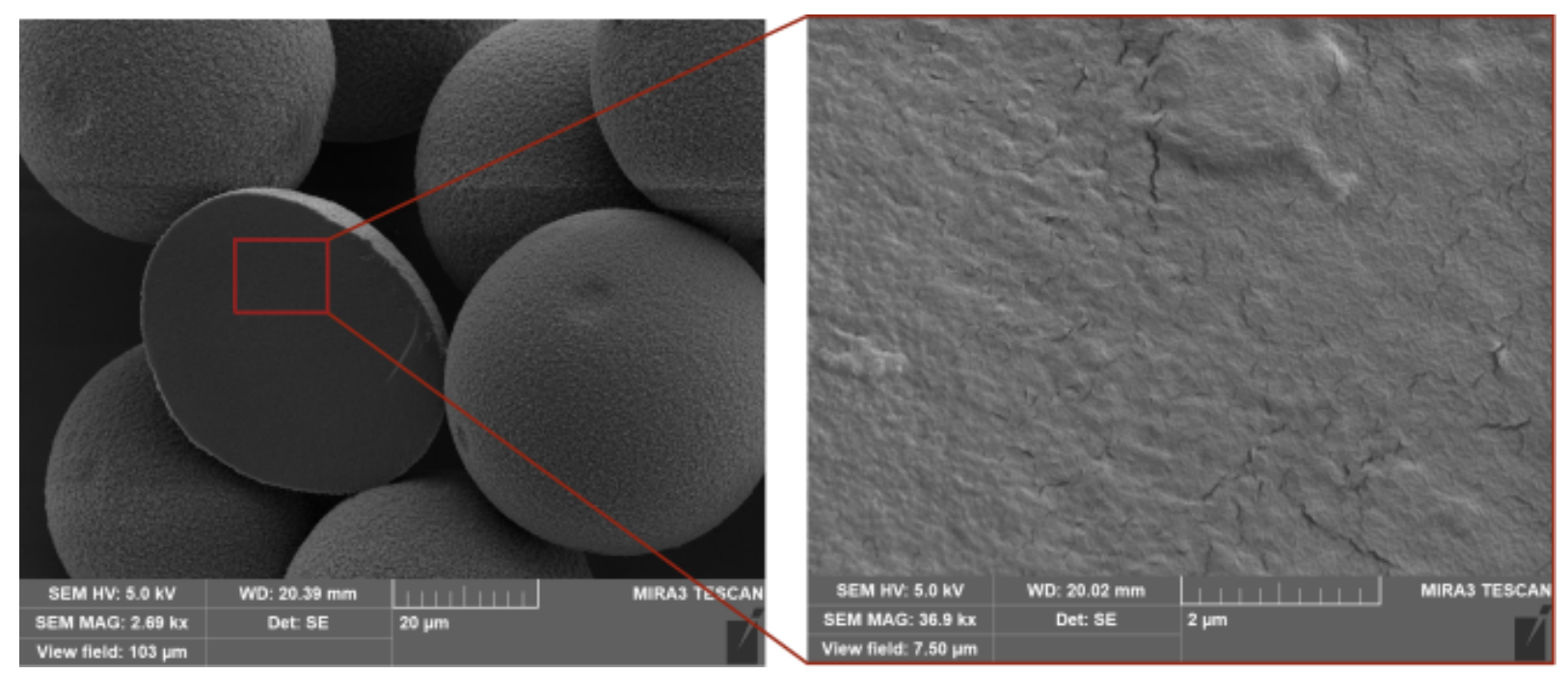

Figure S2: Cross-section of silica microparticle. Silica microparticles made with $1080 \mathrm{mM}$ silicic acid and zoomed in figure from the cross-section.

\section{Fluorescence recovery after photobleaching (FRAP) of Cy5-R5 peptide in silica microparticle}
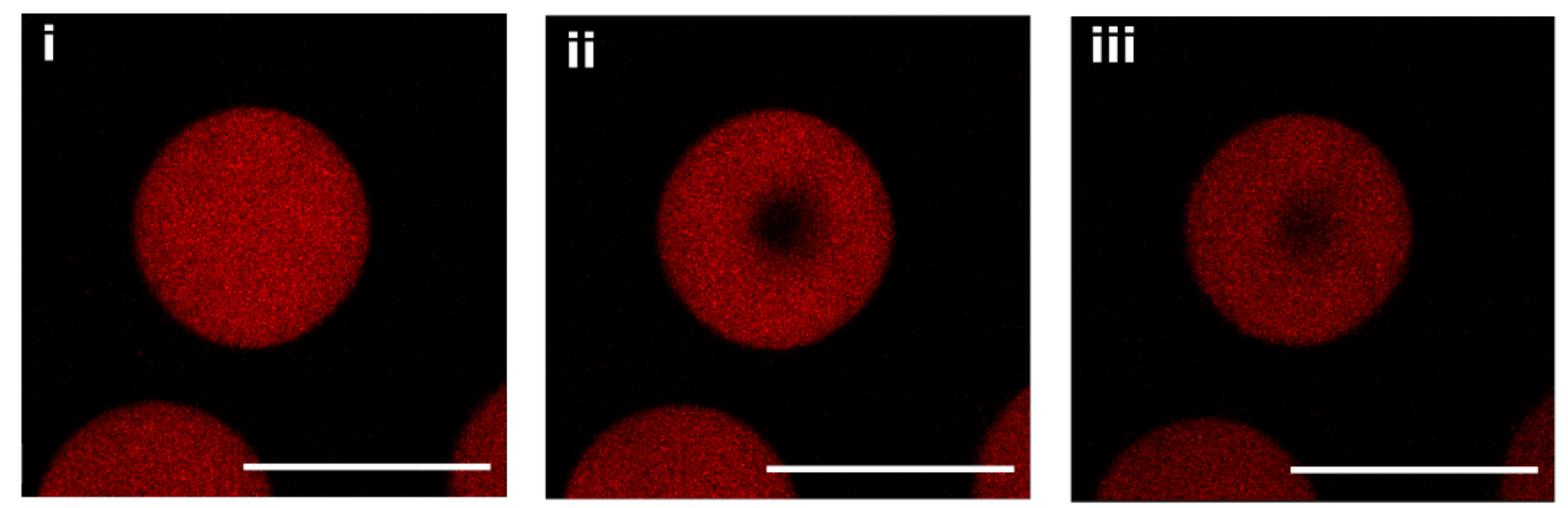

Figure S3: FRAP experiment. Fluorescence recovery after photobleaching (FRAP) experiment was conducted for silica microparticle formed using $1080 \mathrm{mM}$ silicic acid and $1 \mathrm{mg} / \mathrm{ml} \mathrm{Cy} 5$ labelled R5 peptide. Before the FRAP R5 fluorescence is even throughout the microparticle (i). After photobleaching an area from the microparticle (ii) dark spot appeared. The black spot was evident even 3 min after the photobleaching (iii), confirming that the centre of microparticle has also a solid matrix. 


\section{Encapsulation efficiency (EE\%) of FITC-BSA and FITC}

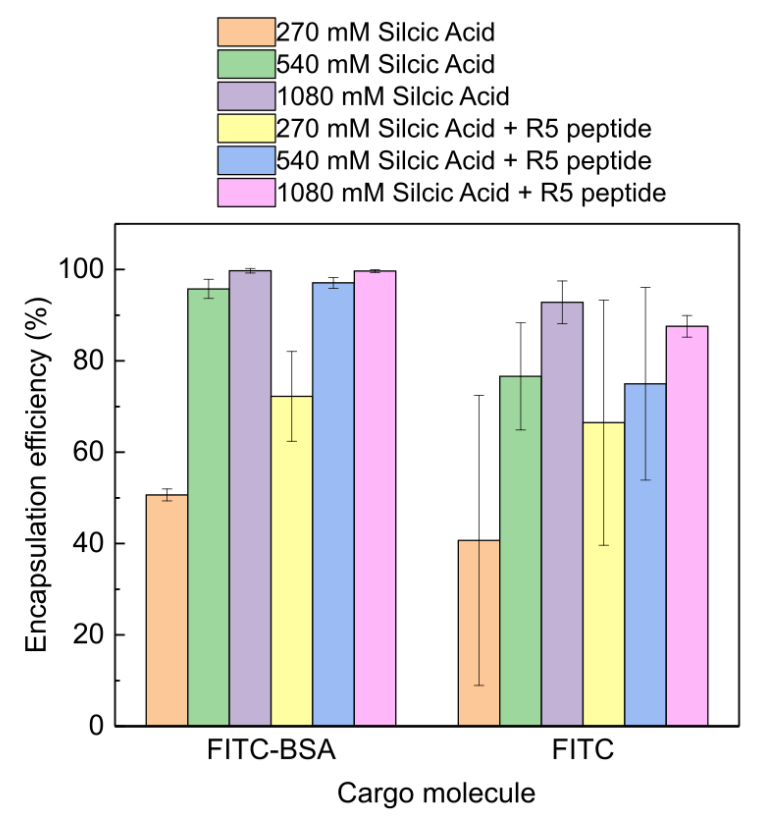

Figure S4: Encapsulation efficiency (EE\%). EE\% of BSA-FITC and FITC into silica microparticles.

Encapsulation efficiency for all used silicic acid conditions were measured (Fig S4) during the kinetic studies. The encapsulation efficiency was calculated using the equation:

$E E \%=\frac{[\text { cargo in }]-[\text { cargo supernatant }]_{\mathrm{t} 0}}{[\text { cargo in }]}$,

Where [cargo in] means the concentration of cargo put in the microparticles and [cargo supernatant $]_{\mathrm{t} 0}$ means the concentration of cargo found at the supernatant right after the emulsification. The encapsulation efficiency was high for all FITC-BSA cases except the lowest silicic acid concentration. However, FITC being considerably smaller, also its encapsulation efficiency varied much more between different microparticle formulations. 


\section{Operation of microfluidic reactor}
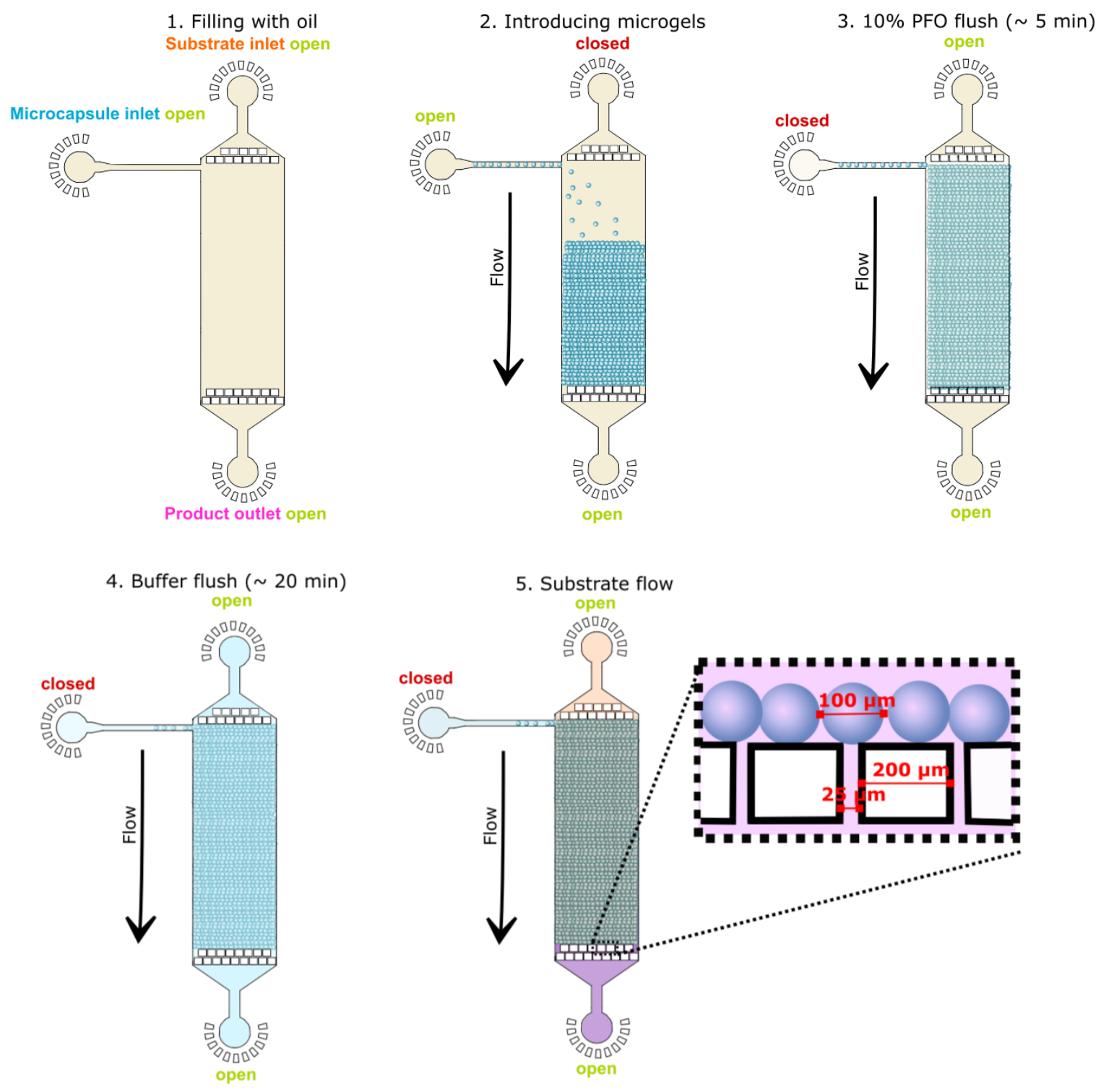

Figure S5: Operation of microfluidic reactor. Step required for filling and operation of the microfluidic reactor with silica microparticles. 
Microfluidic reactor (Fig. S1) with substrate inlet, microparticle inlet and product outlet were designed with AutoCAD so that microparticles could be immobilised and tightly packed. This was possible by adding $25 \mu \mathrm{m}$ filters, which allow liquid flow through the reactor while keeping the $100 \mu \mathrm{m}$ sized microparticles inside. The dimensions of the reactor are: $2 \mathrm{~mm}$ width and $5.9 \mathrm{~mm}$ length.

Microparticles were packed while in oil and the de-emulsification was done inside the device. First the device was filled with oil keeping all the inlets and outlet open. Next, the substrate inlet was closed placing a steel pin inside the inlet followed by insertion of microparticles (in oil). After the whole reactor volume was full of microparticles, the microparticle inlet was closed and $10 \% \mathrm{PFO}$ solution was flown through the device from substrate outlet approximately for $5 \mathrm{~min}$. Now, the oil could be replaced by flushing with water approximately $20 \mathrm{~min}$ or until no oil was evident. Finally, the substrate flow could be started and experiment started.

\section{Reaction time calculations of microfluidic reactor for one enzyme $\beta$-gal and two enzyme GOx/HRP systems}

Microfluidic reactor with volume of $1.40 E-09 \mathrm{~m}^{3}$ was used to pack enzyme silica micro particles into a tight array. In order to obtain kinetic information $\left(K_{m}\right)$ of the encapsulated enzymes, calculations of reaction times were conducted. This reaction time can be then used calculate to reaction rate $\mathrm{v}(\mu \mathrm{M} / \mathrm{min})$ knowing the amount of product (resorufin) at the detection region of the device. This was done transforming the measured product fluorescence (resorufin in both systems) to a concentration $(\mu \mathrm{M})$ by using a calibration curve measured with free resorufin (Fig. S7).

The substrate has time to be transformed to the product in the reactor depending on the used flow. The calculated reaction times (s) are summarised in Table S1. For quantifying the Michelis-Menten kinetics of our device $500 \mu \mathrm{L} / \mathrm{h}$ flow rate was chosen for $\beta$-gal and $31 \mu \mathrm{L} / \mathrm{h}$ for GOx/HRP system. These flow rates were chosen from yield data (Fig. S6b and 6e), because both values lie in the region where the increase of yield seems to be linear against the flow rate/time. 
Table S1: Calculated reaction times inside the microfluidic reactor, for different flow rates

\begin{tabular}{|l|l|}
\hline Flow Rates $(\mu \mathrm{l} / \mathrm{h})$ & Reaction Time $(\mathrm{s})$ \\
\hline 15.625 & $3.23 \mathrm{E}+02$ \\
\hline 31.25 & $1.61 \mathrm{E}+02$ \\
\hline 62.5 & $8.06 \mathrm{E}+01$ \\
\hline 125 & $4.03 \mathrm{E}+01$ \\
\hline 250 & $2.02 \mathrm{E}+01$ \\
\hline 500 & $1.01 \mathrm{E}+01$ \\
\hline
\end{tabular}

\section{Bulk reaction rate of two enzyme GOx/HRP system in bulk}

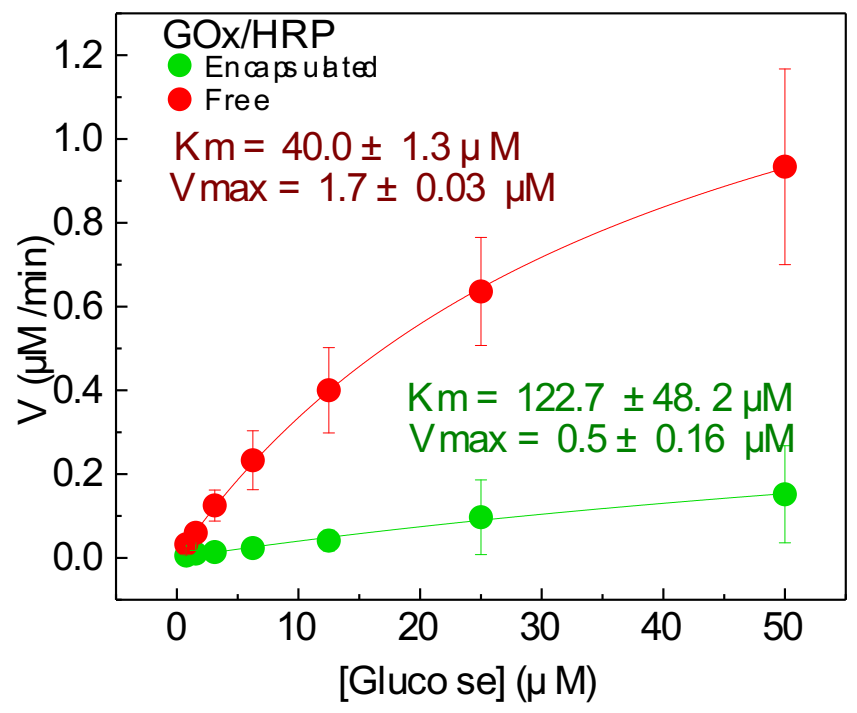

Figure S6: Michaelis-Menten kinetics of GOx/HRP system free and encapsulated into microparticles.

Encapsulating GOx/HRP system into the microparticles has less negative effect in the bulk effect on enzyme kinetics compared to the single enzyme, $\beta$-gal, system. This is due to the fact that it is beneficial to have the enzymes involved in the cascade on close proximity. However, the substrate has to still travel from bulk into the microparticle, which contributes to the slower reaction rate. 


\section{Microfluidic reactor fluorescence intensity}

The product fluorescence in function of substrate concentration shows linear response in both systems examined (Fig. 4). The intensity was monitored from the end of reactor device at the detection area (see article Fig. 5). Background intensity with buffer was measured for each device and subtracted.
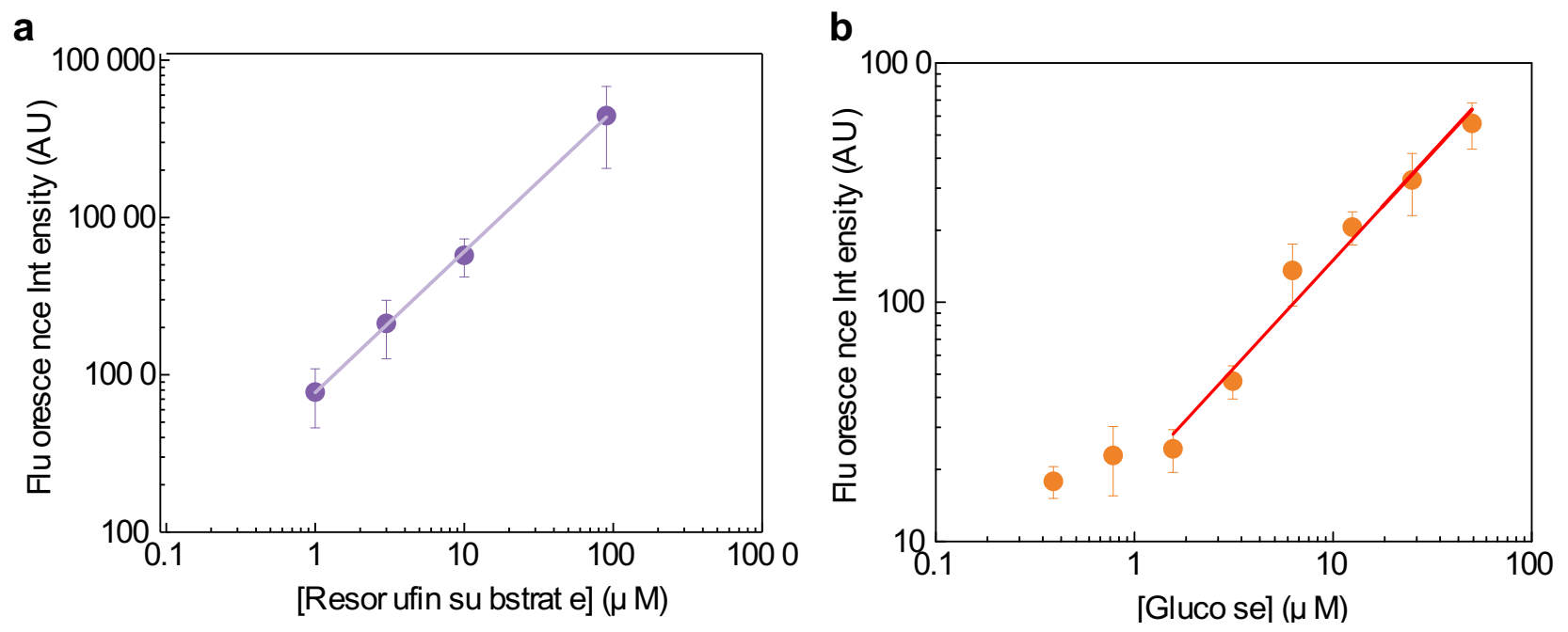

Figure S7: Substrate response of microfluidic reactor. Fluorescence intensity in microfluidic reactor with different substrate concentrations for $\beta$-gal (a) and GOx/HRP (b). Flow rate was kept at $31 \mu \mathrm{L} / \mathrm{h}$ for each sample. 